\title{
On an approximation property of Pisot numbers II
}

\author{
par TOUFIK ZAÏMI
}

\begin{abstract}
RÉSUMÉ. Soit $q$ un nombre complexe, $m$ un entier positif et $l_{m}(q)=\inf \left\{|P(q)|, P \in \mathbb{Z}_{m}[X], P(q) \neq 0\right\}$, où $\mathbb{Z}_{m}[X]$ désigne l'ensemble des polynômes à coefficients entiers de valeur absolue $\leqslant m$. Nous déterminons dans cette note le maximum des quantités $l_{m}(q)$ quand $q$ décrit l'intervalle ] $m, m+1$ [. Nous montrons aussi que si $q$ est un nombre non-réel de module $>1$, alors $q$ est un nombre de Pisot complexe si et seulement si $l_{m}(q)>0$ pour tout $m$.
\end{abstract}

ABSTRACT. Let $q$ be a complex number, $m$ be a positive rational integer and $l_{m}(q)=\inf \left\{|P(q)|, P \in \mathbb{Z}_{m}[X], P(q) \neq 0\right\}$, where $\mathbb{Z}_{m}[X]$ denotes the set of polynomials with rational integer coefficients of absolute value $\leqslant m$. We determine in this note the maximum of the quantities $l_{m}(q)$ when $q$ runs through the interval ] $m, m+1$ [. We also show that if $q$ is a non-real number of modulus $>1$, then $q$ is a complex Pisot number if and only if $l_{m}(q)>0$ for all $m$.

\section{Introduction}

Let $q$ be a complex number, $m$ be a positive rational integer and $l_{m}(q)=$ $\inf \left\{|P(q)|, P \in \mathbb{Z}_{m}[X], P(q) \neq 0\right\}$, where $\mathbb{Z}_{m}[X]$ denotes the set of polynomials with rational integer coefficients of absolute value $\leqslant m$ and not all 0 . Initiated by P. Erdos et al. in [6], several authors studied the quantities $l_{m}(q)$, where $q$ is a real number satisfying $1<q<2$. The aim of this note is to extend the study for a complex number $q$. Mainly we determine in the real case the maximum ( resp. the infimum ) of the quantities $l_{m}(q)$ when $q$ runs through the interval $] m, m+1[$ ( resp. the set of Pisot numbers in ]$m, m+1$ [ ). For the non-real case, we show that if $q$ is of modulus $>1$ then $q$ is a complex Pisot number if and only if $l_{m}(q)>0$ for all $m$. Recall that a Pisot number is a real algebraic integer $>1$ whose conjugates are of modulus $<1$. A complex Pisot number is a non-real algebraic integer of modulus $>1$ whose conjugates except its complex conjugate are of

Manuscrit reçu le 5 septembre 2002. 
modulus $<1$. Note also that the conjugates, the minimal polynomial and the norm of algebraic numbers are considered here over the field of rationals. The set of Pisot numbers ( resp. complex Pisot numbers ) is usually noted $S$ ( resp. $S_{c}$ ). Let us now recall some known results for the real case.

THEOREM A. ( [5], [7] and [9] )

(i) If $q \in] 1, \infty\left[\right.$, then $q$ is a Pisot number if and only if $l_{m}(q)>0$ for all $m$;

(ii) if $q \in] 1,2\left[\right.$, then for any $\varepsilon>0$ there exists $P \in \mathbb{Z}_{1}[X]$ such that $|P(q)|<\varepsilon$.

THEOREM B. ([15])

(i) If $q$ runs through the set $S \cap] 1,2\left[\right.$, then $\inf l_{1}(q)=0$;

(ii) if $m$ is fixed and $q$ runs through the interval $] 1,2\left[\right.$, then $\max l_{m}(q)=$ $l_{m}(A)$, where $A=\frac{1+\sqrt{5}}{2}$.

The values of $l_{m}(A)$ have been determined in [11].

In [3] P. Borwein and K. G. Hare gave an algorithm to calculate $l_{m}(q)$ for any Pisot number $q$ ( or any real number $q$ satisfying $l_{m}(q)>0$ ). The algorithm is based on the following points :

(i) From Theorem A (i), the set $\Omega(q, \varepsilon)=\cup_{d \geqslant 0} \Omega_{d}(q, \varepsilon)$, where $\varepsilon$ is a fixed positive number and

$$
\Omega_{d}(q, \varepsilon)=\left\{|P(q)|, P \in \mathbb{Z}_{m}[X], \partial P=d, 0<|P(q)|<\varepsilon\right\},
$$

is finite ( $\partial P$ is the degree of $P$ );

(ii) if $P \in \mathbb{Z}_{m}[X]$ and satisfies $|P(q)|<\frac{m}{q-1}$ and $\partial P \geqslant 1$, then $P$ can be written $P(x)=x Q(x)+P(0)$ where $Q \in \mathbb{Z}_{m}[X]$ and $|Q(q)|<\frac{m}{q-1}$;

(iii) if $q \in] 1, m+1\left[\right.$, then $1 \in \Omega\left(q, \frac{m}{q-1}\right)$ and $l_{m}(q)$ is the smallest element of the set $\Omega\left(q, \frac{m}{q-1}\right)$ (if $\left.q \in\right] m+1, \infty[$, then from Proposition 1 below we have $\left.l_{m}(q)=1\right)$.

The algorithm consists in determining the sets $\Omega_{d}\left(q, \frac{m}{q-1}\right)$ for $d \geqslant 0$ and the process terminates when $\cup_{k \leqslant d} \Omega_{k}\left(q, \frac{m}{q-1}\right)=\cup_{k \leqslant d+1} \Omega_{k}\left(q, \frac{m}{q-1}\right)$ for some ( the first ) $d$. By (i) a such $d$ exists. In this case, we have $\Omega\left(q, \frac{m}{q-1}\right)=$ $\cup_{k \leqslant d} \Omega_{k}\left(q, \frac{m}{q-1}\right)$ by (ii). For $d=0$, we have $\Omega_{d}\left(q, \frac{m}{q-1}\right)=\{1, \ldots$, $\left.\min \left(m, E\left(\frac{m}{q-1}\right)\right)\right\}$, where $E$ is the integer part function. Suppose that the elements of $\Omega_{d}\left(q, \frac{m}{q-1}\right)$ have been determined. Then, every polynomial $P$ satisfying $|P(q)| \in \Omega_{d+1}\left(q, \frac{m}{q-1}\right)$ is of the form $P(x)=x Q(x)+\eta$, where $|Q(q)| \in \Omega_{d}\left(q, \frac{m}{q-1}\right)$ and $\eta \in\{-m, \ldots 0, \ldots, m\}$. 


\section{The real case}

Let $q$ be a real number. From the definition of the numbers $l_{m}(q)$, we have $l_{m}(q)=l_{m}(-q)$ and $0 \leqslant l_{m+1}(q) \leqslant l_{m}(q) \leqslant 1$, since the polynomial $1 \in \mathbb{Z}_{m}[X]$. Note also that if $q$ is a rational integer ( resp. if $|q|<1$ ), then $l_{m}(q)=1\left(\right.$ resp. $l_{m}(q) \leqslant\left|q^{n}\right|$, where $n$ is a rational integer, and $\left.l_{m}(q)=0\right)$. It follows that without loss of generality, we can suppose $q>1$. The next proposition is a generalization of Remark 2 of [5] and Lemma 8 of [7] :

\section{Proposition 1.}

(i) If $q \in\left[m+1, \infty\left[\right.\right.$, then $l_{m}(q)=1$;

(ii) if $q \in] 1, m+1\left[\right.$, then for any $\varepsilon>0$ there exists $P \in \mathbb{Z}_{m}[X]$ such that $|P(q)|<\varepsilon$.

Proof. (i) Let $q \in\left[m+1, \infty\left[\right.\right.$ and $P(x)=\varepsilon_{0} x^{d}+\varepsilon_{1} x^{d-1}+\ldots+\varepsilon_{d} \in \mathbb{Z}_{m}[X]$, where $d=\partial P \geqslant 1$ ( if $d=0$, then $|P(q)| \geqslant 1$ ). Then,

$$
|P(q)| \geqslant\left|\varepsilon_{0} q^{d}\right|-\left|\varepsilon_{1} q^{d-1}\right|-\ldots-\left|\varepsilon_{d}\right| \geqslant f_{m, d}(q),
$$

where the polynomial $f_{m, d}$ is defined by

$$
f_{m, d}(x)=x^{d}-m\left(x^{d-1}+x^{d-2}+\ldots+x+1\right) .
$$

It suffices now to show that $f_{m, d}(q) \geqslant 1$ and we use induction on $d$. For $d=1$, we have $f_{m, d}(q)=q-m \geqslant m+1-m=1$. Assume that $f_{m, d}(q) \geqslant 1$ for some $d \geqslant 1$. Then, from the recursive formula

$$
f_{m, d+1}(x)=x f_{m, d}(x)-m
$$

and the induction hypothesis we obtain

$$
f_{m, d+1}(q)=q f_{m, d}(q)-m \geqslant q-m \geqslant 1 .
$$

(ii) Let $q \in] 1, m+1\left[\right.$. Then, the numbers $\xi_{j}=\varepsilon_{0}+\varepsilon_{1} q+\ldots+\varepsilon_{n} q^{n}$, where $n$ is a non-negative rational integer and $\varepsilon_{k} \in\{0,1, \ldots, m\}, 0 \leqslant k \leqslant n$ satisfy $0 \leqslant \xi_{j} \leqslant m^{\frac{q^{n+1}-1}{q-1}}$ for all $j \in\left\{1,2, \ldots,(m+1)^{n+1}\right\}$ From the Pigeonhole principle, we obtain that there exist $j$ and $l$ such that $1 \leqslant j<l \leq(m+1)^{n+1}$ and

$$
\left|\xi_{j}-\xi_{l}\right| \leqslant m \frac{q^{n+1}-1}{\left((m+1)^{n+1}-1\right)(q-1)} .
$$

It follows that the polynomial $P \in \mathbb{Z}_{m}[X]$ defined by

$$
P(q)=\xi_{j}-\xi_{l}
$$

satisfies the relation $|P(q)| \leqslant m \frac{q^{n+1}-1}{\left((m+1)^{n+1}-1\right)(q-1)}$ and the result follows by choosing for any $\varepsilon>0$, a rational integer $n$ so that

$$
\frac{m}{(q-1)} \frac{q^{n+1}-1}{(m+1)^{n+1}-1}<\varepsilon .
$$


We cannot deduce from Proposition 1 (ii) that $q$ is an algebraic integer when $q$ satisfies $l_{E(q)}(q)>0$ except for the case $E(q)=1$. However, we have :

Proposition 2. If $l_{E(q)+1}(q)>0$, then $q$ is a beta-number.

Proof. Let $\sum_{n \geqslant 0} \frac{\varepsilon_{n}}{q^{n}}$ be the beta-expansion of $q$ in basis $q$ [13]. Then, $q$ is said to be a beta-number if the subset $\left\{F_{n}(q), n \geqslant 1\right\}$ of the interval [0,1[, where

$$
F_{n}(x)=x^{n}-\varepsilon_{0} x^{n-1}-\varepsilon_{1} x^{n-2}-\ldots-\varepsilon_{n-1},
$$

is finite [12]. Here, the condition $l_{E(q)+1}(q)>0$, implies trivially that $q$ is a beta-number ( as in the proof of Lemma 1.3 of [9] ), since otherwise for any $\varepsilon>0$ there exists $n$ and $m$ such that $n>m, 0<\left|F_{n}(q)-F_{m}(q)\right|<\varepsilon$ and $\left(F_{n}-F_{m}\right) \in \mathbb{Z}_{E(q)+1}[X]$.

Remark 1. Recall that beta-numbers are algebraic integers, Pisot numbers are beta-numbers, beta-numbers are dense in the interval $] 1, \infty[$ and the conjugates of a beta-number $q$ are all of modulus $<\min \left(q, \frac{1+\sqrt{5}}{2}\right)$ ( [4], [12] and [14]). Note also that it has been proved in [8], that if $\left.q \in] 1, \frac{1+\sqrt{5}}{2}\right]$ and $l_{E(q)+1}(q)>0$, then $q \in S$. The question whether Pisot numbers are the only numbers $q>1$ satisfying $l_{E(q)}(q)>0$, has been posed in [7] for the case $E(q)=1$.

From Proposition 1 ( resp. Theorem B ) we deduce that $\inf l_{m}(q)=0$ ( resp. $\left.\max l_{1}(q)=l_{1}(A)\right)$ if $q$ runs through the set $\left.S \cap\right] 1, m+1[$ ( resp. the interval $] 1,2\left[\right.$ ). Letting $A=A_{1}$, we have more generally :

\section{Theorem 1.}

(i) If $q$ runs through the set $S \cap] m, m+1\left[\right.$, then $\inf l_{m}(q)=0$;

(ii) if $q$ runs through the interval $] m, m+1\left[\right.$, then $\max l_{m}(q)=l_{m}\left(A_{m}\right)=$ $A_{m}-m$, where $A_{m}=\frac{m+\sqrt{m^{2}+4 m}}{2}$.

Proof. (i) Let $q \in S \cap] m, m+1$ [, such that its minimal polynomial $P \in$ $\mathbb{Z}_{m}[X]$. Suppose moreover, that there exists a polynomial $Q \in \mathbb{Z}[X]$ satisfying $Q(q)>0$ and $|Q(z)|<|P(z)|$ for $|z|=1$ ( choose for instance $q=A_{m}$ since $m<A_{m}<m+1, P(x)=x^{2}-m x-m$ and $Q(x)=x^{2}-1$. In this case $|P(z)|^{2}-|Q(z)|^{2}=2 m^{2}-1+m(m-1)\left(z+\frac{1}{z}\right)-(m-1)\left(z^{2}+\frac{1}{z^{2}}\right)$ and $\left.|P(z)|^{2}-|Q(z)|^{2} \geqslant 2 m^{2}-1-2 m(m-1)-2(m-1)=1>0\right)$.

From Rouché's theorem, we have that the roots of the polynomial

$$
Q_{n}(x)=x^{n} P(x)-Q(x),
$$

where $n$ is a rational integer $\geqslant \partial P$, are all of modulus $<1$ except only one root, say $\theta_{n}$. Moreover, since $Q_{n}(q)<0$, we deduce that $\theta_{n}>q$ and $\theta_{n}$ $\in S$. 
Now, from the equation

$$
\theta_{n}^{n} P\left(\theta_{n}\right)-Q\left(\theta_{n}\right)=0
$$

we obtain

$$
\left|P\left(\theta_{n}\right)\right|=\frac{\left|Q\left(\theta_{n}\right)\right|}{\theta_{n}^{n}} \leqslant \frac{C_{Q}}{\theta_{n}^{n-\partial Q}} \leqslant \frac{C_{Q}}{q^{n-\partial Q}},
$$

where $C_{Q}$ is a constant depending only on the polynomial $Q$. As $q$ is the only root $>1$ of the polynomial $P$, from the last relation we obtain $\lim \theta_{n}=q$ and $\theta_{n}<m+1$ for $n$ large. Moreover, since $l_{m}\left(\theta_{n}\right) \leqslant\left|P\left(\theta_{n}\right)\right|$, the last relation also yields

$$
\lim l_{m}\left(\theta_{n}\right)=0
$$

and the result follows.

(ii) Note first that $m<A_{m}=\frac{m+\sqrt{m^{2}+4 m}}{2}<m+1$ and $A_{m}^{2}-m A_{m}-m=$ 0 . Let $q \in] m, m+1\left[\right.$ and $q \neq A_{m}$. Then, $l_{m}(q) \leqslant q-m<A_{m}-m$ when $q<A_{m}$. Suppose now $q>A_{m}$ and $l_{m}(q)>0$ ( if $l_{m}(q)=0$, then $\left.l_{m}(q)<A_{m}-m\right)$. Then, from Proposition 1 (ii), we know that for any $\varepsilon>0$, there exists a polynomial $P \in \mathbb{Z}_{m}[X]$ such that $|P(q)|<\varepsilon$. Letting $\varepsilon=l_{m}(q)$, we deduce that there exist a positive rational integer $d$ and $d+1$ elements, say $\eta_{i}$, of the set $\{-m, \ldots, 0, \ldots, m\}$ satisfying $\eta_{0} \eta_{d} \neq 0$ and

$$
\eta_{0}+\eta_{1} q+\ldots+\eta_{d} q^{d}=0
$$

Let $t$ be the smallest positive rational integer such that $\eta_{t} \neq 0$. Then, from the last equation, we obtain

$$
l_{m}(q) \leqslant\left|\eta_{t}+\eta_{t+1} q+\ldots+\eta_{d} q^{d-t}\right|=\left|\frac{\eta_{0}}{q^{t}}\right| \leqslant \frac{m}{q}<\frac{m}{A_{m}}
$$

and

$$
l_{m}(q)<\frac{m}{A_{m}}=A_{m}-m .
$$

To prove the relation $l_{m}\left(A_{m}\right)=A_{m}-m$, we use the algorithm explained in the introduction. With the same notation, we have $\Omega_{0}\left(A_{m}, \frac{m}{A_{m}-1}\right)=\{1\}$, since $\frac{m}{A_{m}-1}=\frac{2 m}{m-2+\sqrt{m^{2}+4 m}}<\frac{5}{3}$. Let $P \in \mathbb{Z}_{m}[X]$. If $\partial P=1$ and $\left|P\left(A_{m}\right)\right| \in$ $\Omega_{1}\left(A_{m}, \frac{m}{A_{m}-1}\right)$, then $P(x)=x-\varepsilon$, where $\varepsilon \in\{-m, \ldots, 0, \ldots, m\}$. A short computation shows that if $\varepsilon \neq m$, then $A_{m}-\varepsilon \geqslant A_{m}-(m-1) \geqslant \frac{m}{A_{m}-1}$. It follows that $\Omega_{1}\left(A_{m}, \frac{m}{A_{m}-1}\right)=\left\{A_{m}-m\right\}$ and if $\partial P=2$ with $\left|P\left(A_{m}\right)\right| \in$ $\Omega_{2}\left(A_{m}, \frac{m}{A_{m}-1}\right)$, then $P(x)=x(x-m)-\varepsilon$. Since $A_{m}\left(A_{m}-m\right)=m$ and the inequality $|m-\varepsilon|<\frac{5}{3}$ holds only for $\varepsilon \in\{m-1, m\}$,we deduce that $P\left(A_{m}\right)= \pm 1, \Omega_{2}\left(A_{m}, \frac{m}{A_{m}-1}\right)=\{1\}, \Omega\left(A_{m}, \frac{m}{A_{m}-1}\right)=\Omega_{0}\left(A_{m}, \frac{m}{A_{m}-1}\right) \cup$ $\Omega_{1}\left(A_{m}, \frac{m}{A_{m}-1}\right)=\left\{1, A_{m}-m\right\}$ and $l_{m}\left(A_{m}\right)=A_{m}-m$.

Corollary. If $q$ runs through the interval $] 1, m+1[$ and is not a rational integer, then $\max l_{m}(q)=l_{m}\left(A_{m}\right)=\frac{2}{1+\sqrt{1+\frac{4}{m}}}$. 
Proof. From the relations $A_{m}=m \frac{1+\sqrt{1+\frac{4}{m}}}{2}$ and $l_{m}\left(A_{m}\right)=\frac{m}{A_{m}}$, we have

$$
\frac{2}{l_{m}\left(A_{m}\right)}=1+\sqrt{1+\frac{4}{m}}>1+\sqrt{1+\frac{4}{m+1}}=\frac{2}{l_{m+1}\left(A_{m+1}\right)}
$$

and the sequence $l_{m}\left(A_{m}\right)$ is increasing with $m$ ( to $\left.1=\lim \frac{2}{1+\sqrt{1+\frac{4}{m}}}\right)$. It follows that $l_{E(q)}\left(A_{E(q)}\right) \leq l_{m}\left(A_{m}\right)$ when $\left.q \in\right] 1, m+1[$. From Theorem 1 (ii), we have $l_{E(q)}(q) \leqslant l_{E(q)}\left(A_{E(q)}\right)$ if $q$ is not a rational integer. Furthermore, since $l_{m}(q) \leqslant l_{E(q)}(q)$ we deduce that $l_{m}(q) \leq l_{m}\left(A_{m}\right)$ and the result follows.

Remark 2. From Theorem B ( resp. Theorem 1 ) we have $\max l_{m+k}(q)=$ $l_{m+k}\left(A_{m}\right)$ when $q$ runs through the interval $] m, m+1[, m=1$ and $k \geqslant 0$ ( resp. $m \geqslant 1$ and $k=0$ ). Recently [1], K. Alshalan and the author considered the case $m=2$ and proved that if $k \in\{1,3,4,5,6\}$ (resp. if $k \in\{2,7,8,9\})$, then $\max l_{2+k}(q)=l_{2+k}(1+\sqrt{2})\left(\operatorname{resp} \cdot \max l_{2+k}(q)=\right.$ $\left.l_{2+k}\left(\frac{3+\sqrt{5}}{2}\right)\right)$.

\section{The non-real case}

Let $a$ be a complex number. As in the real case we have $l_{m}(a)=0$ if $|a|<1$. Since the complex conjugate of $P(a)$ is $P(\bar{a})$ for $P \in \mathbb{Z}_{m}[X]$, we have that $l_{m}(a)=l_{m}(\bar{a})$. Note also that if $a$ is a non-real quadratic algebraic integer and if $P \in \mathbb{Z}_{m}[X]$ and satisfies $P(a) \neq 0$, then $|P(a)| \geqslant 1$, since $|P(a)|^{2}=P(a) P(\bar{a})$ is the norm of the algebraic integer $P(a)$. It follows in this case that $l_{m}(a)=1$.

\section{Proposition 3.}

(i) If $|a| \in\left[m+1, \infty\left[\right.\right.$, then $l_{m}(a)=1$;

(ii) if $|a|^{2} \in[1, m+1[$, then for any positive number $\varepsilon$, there exists $P \in \mathbb{Z}_{m}[X]$ such that $|P(a)|<\varepsilon$.

Proof. (i) The proof is identical to the proof of Proposition 1 (i).

(ii) Let $n \geqslant 0$ be a rational integer and $a^{n}=x_{n}+i y_{n}$, where $x_{n}$ and $y_{n}$ are real and $i^{2}=-1$. Then, the pairs of real numbers

$$
\left(X_{j}, Y_{j}\right)=\left(\varepsilon_{0} x_{0}+\varepsilon_{1} x_{1}+\ldots+\varepsilon_{n} x_{n}, \varepsilon_{0} y_{0}+\varepsilon_{1} y_{1}+\ldots+\varepsilon_{n} y_{n}\right),
$$

where $\varepsilon_{k} \in\{0,1, \ldots, m\}$ for all $k \in\{0,1, \ldots, n\}$, are contained in the rectangle $R=\left[m \sum_{x_{k} \leq 0} x_{k}, m \sum_{0 \leqslant x_{k}} x_{k}\right] \times\left[m \sum_{y_{k} \leq 0} y_{k}, m \sum_{0 \leqslant y_{k}} y_{k}\right]$. If we subdivide each one of two intervals $\left[m \sum_{x_{k} \leqslant 0} x_{k}, m \sum_{0 \leqslant x_{k}} x_{k}\right]$ and $\left[m \sum_{y_{k} \leqslant 0} y_{k}, m \sum_{0 \leqslant y_{k}} y_{k}\right]$ into $N$ subintervals of equal length, then $R$ will be divided into $N^{2}$ subrectangles.

Letting $N=(m+1)^{\frac{n+1}{2}}-1$, where $n$ is odd, then $N^{2}<(m+1)^{n+1}$ and from the pigeonhole principle we obtain that there exist two points 
$\left(X_{j}, Y_{j}\right)$ and $\left(X_{k}, Y_{k}\right)$ in the same subrectangle. It follows that there exist $\eta_{0}, \eta_{1}, \ldots \eta_{n} \in\{-m, \ldots, 0, \ldots, m\}$ not all 0 such that

$$
\begin{aligned}
\left|X_{j}-X_{k}\right| & =\left|\eta_{0} x_{0}+\eta_{1} x_{1}+\ldots+\eta_{n} x_{n}\right| \leqslant \frac{m \sum_{0 \leqslant k \leqslant n}\left|x_{k}\right|}{N}, \\
\left|Y_{j}-Y_{k}\right| & =\left|\eta_{0} y_{0}+\eta_{1} y_{1}+\ldots+\eta_{n} y_{n}\right| \leqslant \frac{m \sum_{0 \leqslant k \leqslant n}\left|y_{k}\right|}{N}
\end{aligned}
$$

and the polynomial $P \in \mathbb{Z}_{m}[X]$ defined by

$$
P(a)=\left(X_{j}-X_{k}\right)+i\left(Y_{j}-Y_{k}\right)=\eta_{0}+\eta_{1} a+\ldots+\eta_{n} a^{n},
$$

satisfies

Since

$$
|P(a)| \leqslant \frac{m}{N} \sqrt{\left(\sum_{0 \leqslant k \leqslant n}\left|x_{k}\right|\right)^{2}+\left(\sum_{0 \leqslant k \leqslant n}\left|y_{k}\right|\right)^{2}} .
$$

( resp.

$$
\max \left(\sum_{0 \leqslant k \leqslant n}\left|x_{k}\right|, \sum_{0 \leqslant k \leqslant n}\left|y_{k}\right|\right) \leqslant \sum_{0 \leqslant k \leqslant n}\left|a^{k}\right|=n+1
$$

$$
\left.\max \left(\sum_{0 \leqslant k \leqslant n}\left|x_{k}\right|, \sum_{0 \leqslant k \leqslant n}\left|y_{k}\right|\right) \leqslant \sum_{0 \leqslant k \leqslant n}\left|a^{k}\right|=\frac{|a|^{n+1}-1}{|a|-1}\right),
$$

when $|a|=1$ ( resp. when $|a|>1$ ), from the last inequality we obtain

$$
|P(a)| \leqslant \frac{m \sqrt{2}}{N}(n+1)
$$

( resp.

$$
\left.|P(a)| \leqslant \frac{m \sqrt{2}}{N} \frac{|a|^{n+1}-1}{|a|-1}\right)
$$

and the result follows by choosing for any $\varepsilon>0$ a rational integer $n$ so that

$$
(m \sqrt{2})\left(\frac{n+1}{\sqrt{(m+1)^{n+1}}-1}\right)<\varepsilon
$$

( resp.

$$
\left.\left(\frac{m \sqrt{2}}{|a|-1}\right)\left(\frac{|a|^{n+1}-1}{\sqrt{(m+1)^{n+1}}-1}\right)<\varepsilon\right) .
$$

Remark 3. The non-real quadratic algebraic integer $a=i \sqrt{m+1}$ satisfies $|a|^{2}=m+1, l_{m}(a)=1$ and is not a root of a polynomial $\in \mathbb{Z}_{m}[X]$, since its norm is $m+1$. Hence, Proposition 3 (ii) is not true for $|a|^{2}=m+1$.

Now we obtain a characterization of the set $S_{c}$.

Theorem 2. Let $a$ be a non-real number of modulus $>1$. Then, $a$ is a complex Pisot number if and only if $l_{m}(a)>0$ for all $m$. 
Proof. The scheme ( resp. the tools ) of the proof is ( resp. are ) the same as in [5] ( resp. in [2] and [10] ) with minor modifications. We prefer to give some details of the proof.

Let $a$ be a complex Pisot number. If $a$ is quadratic, then $l_{m}(a)=1$ for all $m$. Otherwise, let $\theta_{1}, \theta_{2}, \ldots, \theta_{s}$ be the conjugates of modulus $<1$ of $a$ and let $P \in \mathbb{Z}_{m}[X]$ satisfying $P(a) \neq 0$. Then, for $k \in\{1,2, \ldots, s\}$ we have $\left|P\left(\theta_{k}\right)\right| \leqslant m\left(\left|\theta_{k}\right|^{\partial P}+\left|\theta_{k}\right|^{\partial P-1}+\ldots+\left|\theta_{k}\right|+1\right)=m \frac{1-\left|\theta_{k}\right|^{\partial P+1}}{1-\left|\theta_{k}\right|} \leqslant \frac{m}{1-\left|\theta_{k}\right|}$.

Furthermore, since the absolute value of the norm of the algebraic integer $P(a)$ is $\geqslant 1$, the last relation yields

$$
|P(a)|^{2}=|P(a)||P(\bar{a})| \geqslant \frac{\prod_{1 \leqslant k \leqslant s}\left(1-\left|\theta_{k}\right|\right)}{m^{s}}
$$

and

$$
l_{m}(a) \geqslant \sqrt{\frac{\prod_{1 \leqslant k \leqslant s}\left(1-\left|\theta_{k}\right|\right)}{m^{s}}}>0 .
$$

To prove the converse, note first that if $a$ is a non-real number such that $l_{m}(a)>0$ for all $m$, then $a$ is an algebraic number by Proposition 3 (ii). In fact we have :

Lemma 1. Let a be a non-real number of modulus $>1$. If $l_{m}(a)>0$ for all $m$, then $a$ is an algebraic integer.

Proof. As in the proof of Proposition 2, we look for a representation $a=$ $\sum_{n \geqslant 0} \frac{\varepsilon_{n}}{a^{n}}$ of the number $a$ in basis $a$ where the absolute values of the rational integers $\varepsilon_{n}$ are less than a constant $c$ depending only on $a$. In fact from Lemma 1 of [2], such a representation exists with $c=E\left(\frac{1}{2}+\left|a^{2}\right| \frac{|a|+1}{|\sin t|}\right)$, where $a=|a| e^{i t}$. Then, the polynomials

$$
F_{n}(x)=x^{n}-\varepsilon_{0} x^{n-1}-\varepsilon_{1} x^{n-2}-\ldots-\varepsilon_{n-1},
$$

where $n \geqslant 1$, satisfy $F_{n} \in \mathbb{Z}_{c}[X]$ and

$$
\left|F_{n}(a)\right|=\left|\sum_{k \geqslant 0} \frac{\varepsilon_{n+k}}{a^{k+1}}\right| \leqslant \frac{c}{|a|-1} .
$$

It follows that if $l_{2 c}(a)>0$, then the set $\left\{F_{n}(a), n \geqslant 1\right\}$ is finite. Consequently, there exists $n$ and $m$ such that $n>m$ and $F_{n}(a)=F_{m}(a)$, so that $a$ is a root of the monic polynomial $\left(F_{n}-F_{m}\right) \in \mathbb{Z}_{2 c}[X]$.

To complete the proof of Theorem 2 it suffices to prove the next two results. 
Lemma 2. Let a be an algebraic integer of modulus $>1$. If $l_{m}(a)>0$ for all $m$, then a has no conjugate of modulus 1 .

Proof. Let $I_{m}=\left\{F \in \mathbb{Z}_{m}[X], F(x)=P(x) Q(x), Q \in \mathbb{Z}[X]\right\}$, where $P$ is the minimal polynomial of $a$. Let $F \in I_{m}$ and define a sequence $F^{(k)}$ in $\mathbb{Z}_{m}[X]$ by the relations $F^{(0)}=F$ and $F^{(k+1)}(x)=\frac{F^{(k)}(x)-F^{(k)}(0)}{x}$, where $k$ is a non-negative rational integer. Then, the polynomials $F^{(k)}$ satisfy $\left|F^{(k)}(a)\right| \leqslant \frac{m}{|a|-1}$. Indeed, we have $F^{(0)}(a)=0$ and $\left|F^{(k+1)}(a)\right| \leqslant$ $\frac{\left|F^{(k)}(a)\right|+\left|F^{(k)}(0)\right|}{|a|} \leqslant \frac{m}{|a|(|a|-1)}+\frac{m}{|a|}=\frac{m}{|a|-1}$, when $\left|F^{(k)}(a)\right| \leqslant \frac{m}{|a|-1}$. Let $R_{F}^{(k)} \in \mathbb{Z}[X]$ be the remainder of the euclidean division of the polynomial $F^{(k)}$ by $P$. Since $P$ is irreducible and $\partial R_{F}^{(k)}<\partial P$, the set of polynomials $\left\{R_{F}^{(k)}, k \geqslant 0, F \in I_{m}\right\}$ is finite when the complex set $\left\{R_{F}^{(k)}(a), k \geqslant 0\right.$, $\left.F \in I_{m}\right\}$ is finite.

Suppose now that $a$ has a conjugate of modulus 1. Then, from Proposition 2.5 of [10], there exists a positive rational integer $c$ so that the set $\left\{R_{F}^{(k)}, k \geqslant 0, F \in I_{c}\right\}$ is not finite. Hence, the bounded set $\left\{R_{F}^{(k)}(a)=\right.$ $\left.F^{(k)}(a), k \geqslant 0, F \in I_{c}\right\}$ is not finite and for any $\varepsilon>0$, there exist $F_{1} \in I_{c}$ and $F_{2} \in I_{c}$ such that $0<\left|F_{1}^{(k)}(a)-F_{2}^{(j)}(a)\right|<\varepsilon$, where $k$ and $j$ are non-negative rational integers. Hence, $l_{2 c}(a)=0$, and this contradicts the assumption $l_{m}(a)>0$ for all $m$.

Lemma 3. Let a be an algebraic integer of modulus $>1$. If $l_{m}(a)>0$ for all $m$, then a has no conjugate of modulus $>1$ other than its complex conjugate.

Proof. Let $J_{m}$ be the set of polynomials $F \in \mathbb{Z}_{m}[X]$ satisfying $F(a)=$ $\frac{S\left(\frac{1}{a}\right)}{a}$, for some $S \in \mathbb{Z}_{m}[[X]]$ ( the set of formal series with rational integers coefficients of absolute value $\leqslant m)$. If the polynomials $F^{(k)}$ and $R_{F}^{(k)}$ are defined for $F \in J_{m}$ by the same way as in the precedent proof ( $I_{m} \subset J_{m}$ ), we obtain immediately $F^{(k)} \in J_{m}$ and $|F(a)|=\left|\frac{S\left(\frac{1}{a}\right)}{a}\right| \leqslant \frac{m}{|a|-1}$. Therefore, by the previous argument, the set $\left\{R_{F}^{(k)}, k \geqslant 0, F \in J_{m}\right\}$ is finite when $l_{2 m}(a)>0$.

Let $\alpha$ be a conjugate of modulus $>1$ of $a$ and let $S(x)=\sum_{n} s_{n} x^{n} \in$ $\mathbb{Z}_{m}[[X]]$ satisfying $S\left(\frac{1}{a}\right)=0$. Then, $S\left(\frac{1}{\alpha}\right)=0$. Indeed, if $F(x)=s_{0} x^{n}+$ $s_{1} x^{n-1}+\ldots+s_{n}$, then $F \in J_{m}, F(\alpha)=R_{F}^{(0)}(\alpha)$ and

$$
S\left(\frac{1}{\alpha}\right)=\lim \left(s_{0}+\frac{s_{1}}{\alpha}+\ldots+\frac{s_{n}}{\alpha^{n}}\right)=\lim \frac{F(\alpha)}{\alpha^{n}}=\lim \frac{R_{F}^{(0)}(\alpha)}{\alpha^{n}}=0,
$$

since the coefficients of the polynomial $R_{F}^{(0)}$ are bounded $\left(R_{F}^{(0)} \in\left\{R_{F}^{(k)}\right.\right.$, $\left.\left.k \geqslant 0, F \in J_{m}\right\}\right)$. It suffices now to find for $\alpha \notin\{a, \bar{a}\}$ a positive rational 
integer $m$ and an element $S$ of $\mathbb{Z}_{m}[[X]]$ satisfying $S\left(\frac{1}{a}\right)=0$ and $S\left(\frac{1}{\alpha}\right) \neq 0$. In fact this follows from Proposition 7 of [2].

Now from Theorem 1 we have the following analog:

\section{Proposition 4.}

(i) If a runs through the set $S_{c} \cap\{z, \sqrt{m}<|z|<\sqrt{m+1}\}$, then $\inf l_{m}(a)=0$;

(ii) if a runs through the annulus $\{z, \sqrt{m}<|z|<\sqrt{m+1}\}$, then $\sup l_{m}(a) \geqslant l_{m}\left(i \sqrt{A_{m}}\right)=A_{m}-m$.

Proof. First we claim that if $q$ is a real number $>1$, then $l_{m}(q)=l_{m}(i \sqrt{q})$. Indeed, let $P \in \mathbb{Z}_{m}[X]$ such that

$$
P(q)=\eta_{0}+\eta_{1} q+\ldots+\eta_{\partial P} q^{\partial P} \neq 0 .
$$

Then,

$$
P(q)=\eta_{0}-\eta_{1}(i \sqrt{q})^{2}+\ldots \pm \eta_{\partial P}(i \sqrt{q})^{2 \partial P}=Q(i \sqrt{q}),
$$

where $Q \in \mathbb{Z}_{m}[X]$ and $\partial Q=2 \partial P$. It follows that $|P(q)| \geqslant l_{m}(i \sqrt{q})$ and $l_{m}(q) \geqslant l_{m}(i \sqrt{q})$. Conversely, let $P \in \mathbb{Z}_{m}[X]$ such that

$$
P(i \sqrt{q})=\eta_{0}+\eta_{1}(i \sqrt{q})+\eta_{2}(i \sqrt{q})^{2}+\ldots+\eta_{\partial P}(i \sqrt{q})^{\partial P} \neq 0 .
$$

Then, the polynomial $R$ ( resp. $I) \in \mathbb{Z}_{m}[X] \cup\{0\}$ defined by

$$
R(q)=\frac{P(i \sqrt{q})+P(-i \sqrt{q})}{2}=\eta_{0}-\eta_{2} q+\ldots \pm \eta_{2 s} q^{s},
$$

where $0 \leqslant 2 s \leqslant \partial P$, satisfies $|R(q)| \leqslant|P(i \sqrt{q})|$ ( resp.

$$
I(q)=\frac{P(i \sqrt{q})-P(-i \sqrt{q})}{2 i \sqrt{q}}=\eta_{1}-\eta_{3} q+\ldots \pm \eta_{2 t+1} q^{t}
$$

where $0 \leqslant 2 t+1 \leqslant \partial P$, satisfies $\left.|I(q)| \leqslant\left|\frac{P(i \sqrt{q})}{\sqrt{q}}\right|<|P(i \sqrt{q})|\right)$.

Since $P(i \sqrt{q}) \neq 0$, at least one of the quantities $R(q)$ and $I(q)$ is $\neq 0$. It follows that $l_{m}(q) \leqslant|P(i \sqrt{q})|$ and $l_{m}(q) \leqslant l_{m}(i \sqrt{q})$.

Note also that if $q \in S$, then $i \sqrt{q} \in S_{c}$ and conversely if $i \sqrt{q} \in S_{c}$, where $q$ is a real number, then $q \in S$. Hence, by Theorem 1 we have

$$
0 \leqslant \inf l_{m}(a) \leqslant \inf l_{m}(i \sqrt{q})=\inf l_{m}(q)=0,
$$

( resp.

$$
l_{m}\left(i \sqrt{A_{m}}\right)=l_{m}\left(A_{m}\right)=\max l_{m}(q)=\max l_{m}(i \sqrt{q}) \leq \sup l_{m}(a),
$$

when $a$ runs through the set $S_{c} \cap\{z, \sqrt{m}<|z|<\sqrt{m+1}\}$ and $q$ runs through the set $S \cap] m, m+1$ [ ( resp. when $a$ runs through the annulus $\{z, \sqrt{m}<|z|<\sqrt{m+1}\}$ and $q$ runs through the interval $] m, m+1[$ ). 
Remark 4. The question of [7] cited in Remark 1, can also be extended to the non-real case : Are complex Pisot numbers the only non-real numbers $a$ satisfying $l_{E\left(\left|a^{2}\right|\right)}(a)>0, a^{2}+1 \neq 0$ and $a^{2}-a+1 \neq 0$ ?

Acknowledgements. The author wishes to thank the referee for careful reading of the manuscript and K. G. Hare for his remarks.

\section{References}

[1] K. Alshalan and T. ZAIMI, Some computations on the spectra of Pisot numbers. Submitted.

[2] D. Berend and C. Frougny, Computability by finite automata and Pisot Bases. Math. Systems Theory 27 (1994), 275-282.

[3] P. BoRWEIN and K. G. HARE, Some computations on the spectra of Pisot and Salem numbers. Math. Comp. 71 No. 238 (2002), 767-780.

[4] D. W. BoYD, Salem numbers of degree four have periodic expansions. Number Theory (eds J.-H. DE ConincK and C. LevesQue, Walter de Gruyter, Berlin) 1989, 57-64.

[5] Y. Bugeaud, On a property of Pisot numbers and related questions. Acta Math. Hungar. 73 (1996), 33-39.

[6] P. ERDös, I. Joó and V. KomornIK, Characterization of the unique expansions $1=$ $\sum_{i \geqslant 1} q^{-n_{i}}$ and related problems. Bull. Soc. Math. France 118 (1990), 377-390.

[7] P. ERDös, I. Joó and V. KoMORNIK, On the sequence of numbers of the form $\varepsilon_{0}+\varepsilon_{1} q+$ $\ldots+\varepsilon_{n} q^{n} \varepsilon_{i} \in\{0,1\}$. Acta Arith. 83 (1998), 201-210.

[8] P. ERDös, I. Joó and F. J. Schnitzer, On Pisot numbers. Ann. Univ. Sci. Budapest Eotvos Sect. Math. 39 (1996), 95-99.

[9] P. ERDös and V. Komornik, Developments in non integer bases. Acta Math. Hungar. 79 (1998), 57-83.

[10] C. Frougny, Representations of numbers and finite automata. Math. Systems Theory 25 (1992), 37-60.

[11] V. Komornik, P. LoReti and M. Pedicini, An approximation property of Pisot numbers. J. Number Theory 80 (2000), 218-237.

[12] W. PARRY, On the $\beta$-expansions of real numbers. Acta Math. Acad. Sci. Hungar. 11 (1960), 401-416.

[13] A. RÉNYI, Representations for real numbers and their ergodic properties. Acta Math. Hungar. 8 (1957), 477-493.

[14] B. SolOMYAK, Conjugates of beta-numbers and the zero-free domain for a class of analytic functions. Proc. London Math. Soc. 68 (1994), 477-498.

[15] T. ZAїMI, On an approximation property of Pisot numbers. Acta Math. Hungar. 96 (4) (2002), 309-325.

Toufik ZAÏMI

King Saud University

Dept. of Mathematics

P. O. Box 2455

Riyadh 11451, Saudi Arabia

E-mail : zaimitou@ksu.edu.sa 